\title{
Magnetic Dual-template Molecularly Imprinted Polymer Nanoparticles for the Simultaneous Determination of Acetaminophen and Codeine in Urine Samples by Ion Mobility Spectrometry
}

\author{
Mohammad T. JAFARI, ${ }^{\dagger}$ Behzad REzAEI, and Hajar BAHRAMI \\ Department of Chemistry, Isfahan University of Technology, Isfahan 84156-83111, Iran
}

\begin{abstract}
Dual-template magnetic molecularly imprinted polymer nanoparticles were synthesized and used for the solid-phase extraction of acetaminophen and codeine before simultaneous determination by corona discharge ion mobility spectrometry. The magnetic molecularly imprinted polymer nanoparticles were prepared using silica-coated magnetic nanoparticles as supporters, acetaminophen and codeine as template molecules, 3-aminopropyltriethoxysilane and phenyltriethoxysilane as functional monomers, and tetraethoxysilane as a cross-linker. The obtained molecularly imprinted polymer was characterized by transmission electron microscopy, x-ray diffraction and Fourier-transform infrared spectroscopy. The adsorption performance of the imprinted polymers was studied by a series of experiments, indicating a satisfactory recognition ability of products for acetaminophen and codeine. The detection limits of 0.05 and $0.12 \mu \mathrm{g} \mathrm{mL} L^{-1}$, and the dynamic range of $0.20-2.0$ and $0.40-3.0 \mu \mathrm{g} \mathrm{mL} \mathrm{m}^{-1}$ were achieved for acetaminophen and codeine, respectively. The proposed method was used for simultaneous determinations of acetaminophen and codeine in urine samples, and the corresponding recoveries were calculated in the range of $87-94 \%$. These satisfactory results revealed the ability of the method for a routine analysis of acetaminophen and codeine, simultaneously.
\end{abstract}

Keywords Dual-template, magnetic molecularly imprinted polymer, nanoparticles, ion mobility spectrometry

(Received September 4, 2017; Accepted October 10, 2017; Published March 10, 2018)

\section{Introduction}

Acetaminophen or paracetamol ( $4^{\prime}$-hydroxyacetanilide) is known as a painkiller and antipyretic drug and used for moderate pain as well as for lowering fever. ${ }^{1}$ It is the main ingredient in numerous flu and cold medications. Acetaminophen is used to relieve terrible pain, such as cancer pain and after surgery, in combination with an opioid pain drug. ${ }^{2,3}$

Codeine is often obtained synthetically, and is available in salt form. This compound is used as an effectual painkiller and antitussive factor in medical preparations. ${ }^{4,5}$ Whereof codeine belongs to the narcotic category, whose more important effects are on the nervous system, it can generate psychological dependence. ${ }^{6}$

Combinations of codeine with acetaminophen create efficient pain relief with reduced side effects. The combination causes effectual pain relief by about $40 \%$ more than that offered by the acetaminophen alone. ${ }^{7}$ Quality control of these drugs are important because of complications related to a drug overdose. Since acetaminophen metabolism is mainly in the liver, overdoses of acetaminophen can cause liver damage. This drug also causes renal insufficiency. ${ }^{8}$ High doses of codeine is also causing coma and depression. ${ }^{9}$

There are various methods for the simultaneous identification

$\dagger$ To whom correspondence should be addressed.

E-mail: jafari@cc.iut.ac.ir of acetaminophen and codeine, such as high-performance liquid chromatography (HPLC), ${ }^{2,10}$ spectrophotometry, ${ }^{11,12}$ chemiluminescence, ${ }^{13}$ and electrochemical methods. ${ }^{14-16}$ With the purpose of analytes detection, more efficiently, the pretreatment of sample matrices with various methods, such as solid/liquid phase extraction, is beneficial to increase the analysis accuracy. ${ }^{17}$ So far, one of the methods that has mostly been used for the extraction and preconcentration of analytes is solid-phase extraction (SPE). A rather new development in this field is utilizing the molecularly imprinted polymers, helping us to obtain an efficient selective extraction. ${ }^{18,19}$

Molecularly imprinting, firstly introduced by Wulff, ${ }^{20}$ has specific three-dimensional identification sites supplementary to the size and shape of the template molecules. This leads to functionality interaction between the imprinted site and template. $^{21,22}$ A conventional method for the synthesis of molecularly imprinted polymers (MIPs) is bulk polymerization, ${ }^{23,24}$ representing good selectivity. However, this method has disadvantages, such as heterogeneous dispensation of the binding sites, poor site approachability for template molecule and imperfect template elimination. ${ }^{25}$ In order to dominate these drawbacks, the surface molecularly imprinting technique has been expanded. Hereof, surface grafted MIPs have been applied on the various surfaces, such as silica particles, ${ }^{26}$ polymeric supports, ${ }^{27}$ titanium dioxide particles ${ }^{28}$ and magnetic nanoparticles (MNPs). ${ }^{29,30}$ The MNPs possess significant advantages, such as small size, high surface-tovolume ratio, and easy separation from extraction system using 
a magnetic field. ${ }^{31}$ Thus, a combination of magnetic properties of MNPs and advantages of MIP shows high selectivity for the target molecule, so it provides the capability of one-step separation. ${ }^{29}$ On the other hand, some of the advantages of multiple MIP compared with single MIP are the simultaneous extraction of analytes in one process, lower solvent consumption, cost effectiveness, and the short analysis time. In fact, multitemplate MIP reduces the efforts needed to prepare different MIPs for different analytes, separately. There are various detection systems (e.g. quartz crystal microbalance devices and surface plasmon resonance spectroscopy) used after the separation of compounds by MIPs. In 2009, ${ }^{32}$ we introduced ion mobility spectrometry (IMS) as a powerful technique for the analysis of analyte extracted by MIPs. Some other works ${ }^{33-36}$ were performed using MIP-IMS, and satisfactory results revealed the capability of this combination for the analysis of some drugs in different matrices.

Briefly, IMS is an analytical technique used to separate and detect gas-phase ions based on their mobility in a constant electric field. IMS is a sensitive technique for detecting chemical and biological agents, such as drugs, explosives, illicit drugs, pesticides, oligonucleotides and peptides based on the movement of ions in the gas phase, under the influence of an electric field. ${ }^{37}$ In this way, the separation of generated ions is based on differences in their intrinsic mobility in an electric field, and the mobility of ions is dependent on the molecular weight, size and structure of ions. The significant features of this method are a very low detection limit, a rapid response and low cost as well as miniaturization and portability. In addition, this technique can be used as detection system for gas and liquid chromatography, supercritical fluid and electrophoresis method. This technique has some advantages compared to other instruments such as, HPLC, GC, and mass spectrometry. For example, IMS needs no expensive solvents, requires no vacuum, has a short detection time, and finally with this technique it is not necessary to derivatize the compound. However, the lack of specificity is the main disadvantages in IMS technique. In fact, the separation of ions occurs at atmospheric pressure and therefore the random collisions broaden peaks, making spectral overlapping. To overcome this problem, it is necessary to use a powerful method of extraction and clean-up, before sample analysis by IMS.

In this research, we synthesized magnetic dual-template molecularly imprinted polymers (Dual-MIPs) combining the sol-gel approach, surface imprinting technique, and magnetic separation for the simultaneous determinations of acetaminophen and codeine by corona discharge ion mobility spectrometry. The characterization and adsorption efficiency of magnetic molecularly imprinted polymers (MMIPs) such as isothermal and kinetic adsorptions, selectivity study, repeatability and reusability were studied in details. Finally, the method was used for the analysis of urine sample.

\section{Experimental}

\section{Materials}

Acetaminophen was obtained from Amin Pharmaceutical Co (Isfahan, Iran). Codeine was prepared from Temad Pharmaceutical Co (Tehran, Iran). Ethanol (99.6 - 99.9\%) was purchased from Bidestan (Qazvin, Iran). Ferrous chloride tetrahydrate $\left(\mathrm{FeCl}_{2} \cdot 4 \mathrm{H}_{2} \mathrm{O},>99 \%\right)$ and ferric chloride hexahydrate $\left(\mathrm{FeCl}_{3} \cdot 6 \mathrm{H}_{2} \mathrm{O}\right.$, >99\%) were purchased from Riedel-de-Haen (Seelze, Germany). Ammonia solution (25\%,w/w) and 2-propyl alcohol were purchased from Merck (Darmstadt, Germany).
Tetraethoxysilane (TEOS), 3-aminopropyltriethoxysilane (APTES), and phenyltriethoxysilane (PTEOS) were prepared from Sigma-Aldrich (Steinheim, Germany).

\section{Instrumentation}

The corona discharge ionization ion mobility spectrometer (CDIMS) used in this work was manufactured at Isfahan University of Technology. The details were mentioned in our previous article. $^{38}$ The operational conditions of CD-IMS are given in Table S1 (Supporting Information). MMIPs were characterized by transmission electron microscopy (TEM) (Carl Zeiss SMT, USA), Fourier transform infrared spectroscopy (FT-IR) Model 350 (Jasco, Tokyo, Japan) and X-ray diffraction (XRD) (Philips, Model XPERT).

\section{Synthesis of $\mathrm{Fe}_{3} \mathrm{O}_{4} \mathrm{MNPS}$}

$\mathrm{Fe}_{3} \mathrm{O}_{4}$ nanoparticles were created based on a previous method described by Wang et al. ${ }^{29}$ To this end, $1.72 \mathrm{~g} \mathrm{FeCl}_{2} \cdot 4 \mathrm{H}_{2} \mathrm{O}$ and $4.72 \mathrm{~g} \mathrm{FeCl}_{3} \cdot 6 \mathrm{H}_{2} \mathrm{O}$ was dissolved in deionized water $(80 \mathrm{~mL})$ with intensive stirring ( $800 \mathrm{rpm}$ ), degassed with nitrogen gas for $15 \mathrm{~min}$ and heated to $80^{\circ} \mathrm{C}$. Afterward, $10 \mathrm{~mL}$ of ammonium hydroxide $(25 \%)$ was added to the solution and the reaction was performed for $30 \mathrm{~min}$. The product was isolated by a magnet and rinsed six times with deionized water to eliminate any nonereacted chemicals, and finally dried under a vacuum.

\section{Preparation of $\mathrm{Fe}_{3} \mathrm{O}_{4} @ \mathrm{SiO}_{2}$}

The synthesized $\mathrm{Fe}_{3} \mathrm{O}_{4}$ nanoparticles $(300.0 \mathrm{mg})$ were dispersed in $4 \mathrm{~mL}$ of deionized water and $50 \mathrm{~mL}$ 2-propyl alcohol and sonicated for $15 \mathrm{~min}$. Afterward, $5 \mathrm{~mL}$ of ammonium hydroxide and $2 \mathrm{~mL}$ of tetraethoxysilane were added to the suspension. The reaction was carried out for $12 \mathrm{~h}$, and finally, the product was isolated by a magnet and washed with deionized water for six times, before drying under a vacuum. ${ }^{29}$

\section{Synthesis of $\mathrm{Fe}_{3} \mathrm{O}_{4} @$ Dual-MIPs}

For the preparation of $\mathrm{Fe}_{3} \mathrm{O}_{4} @$ Dual-MIPs, acetaminophen $(20 \mathrm{mg})$ and codeine $(20 \mathrm{mg})$ were dissolved in ethanol $(20 \mathrm{~mL})$. Then, $110 \mu \mathrm{L}$ of APTES and $220 \mu \mathrm{L}$ of PTEOS were added to the mixture and was stirred for $2 \mathrm{~h}$. Afterward, $0.2 \mathrm{~g}$ of $\mathrm{Fe}_{3} \mathrm{O}_{4} @$ $\mathrm{SiO}_{2}, 600 \mu \mathrm{L}$ of ammonium hydroxide, and $400 \mu \mathrm{L}$ of TEOS were added to the mixture and the reaction performed for $6 \mathrm{~h}$ under continuous stirring. The reaction was allowed to be complete and then the products were washed with deionized water to neutralize the supernatant liquid. The solution of methanol-acetic acid $(9: 1, \mathrm{v} / \mathrm{v})$ was then added to eliminate the templates. Then, the product was rinsed with deionized water and dried in the vacuum. The mass of obtained MIP was $0.32 \mathrm{~g}$ indicating the ratio of 0.625 for $\mathrm{Fe}_{3} \mathrm{O}_{4} @ \mathrm{SiO}_{2}$-MIP. The $\mathrm{Fe}_{3} \mathrm{O}_{4} @$ NIP was prepared by the same method, however, in the lack of templates. ${ }^{39}$

\section{Sorption capacity}

To survey the adsorption capacity of the $\mathrm{Fe}_{3} \mathrm{O}_{4} @$ Dual-MIPs and $\mathrm{Fe}_{3} \mathrm{O}_{4} @ \mathrm{NIPs}$, the kinetic and isothermal adsorptions were investigated. In order to study kinetic adsorption, $10 \mathrm{mg}$ $\mathrm{Fe}_{3} \mathrm{O}_{4} @$ Dual-MIPs and $10 \mathrm{mg} \mathrm{Fe}_{3} \mathrm{O}_{4} @$ NIPs were added to $10 \mathrm{~mL}$ methanol solutions of acetaminophen $\left(20 \mu \mathrm{g} \mathrm{mL}^{-1}\right)$ and codeine $\left(15 \mu \mathrm{g} \mathrm{mL}^{-1}\right)$ and then shaken for defined time periods $(10-100 \mathrm{~min})$. The supernatant and MIP were separated by a magnet and the concentration of acetaminophen and codeine in supernatant liquid were measured by CD-IMS. Adsorption capacity $\left(Q, \mathrm{mg} \mathrm{g}^{-1}\right)$ was also calculated by the following equation: 


$$
Q=\frac{\left(C_{0}-C_{1}\right) V}{W}
$$

Here, $C_{0}$ and $C_{1}$ are the initial and final concentrations of the templates in $\mathrm{mg} \mathrm{L}^{-1}$, respectively, $V(\mathrm{~L})$ is the initial solution volume, and $W(\mathrm{~g})$ is the sorbent weight.

In order to study the capacity of isothermal adsorption for the sorbent, the experiments were carried out by placing $10 \mathrm{mg}$ of the imprinted polymer and $10 \mathrm{~mL}$ of different concentrations of acetaminophen and codeine in the range of 5.0 to 35.0 and 5.0 to $30.0 \mu \mathrm{g} \mathrm{mL}^{-1}$, respectively, for $30 \mathrm{~min}$. Then, the sorbents were separated by a magnet and the concentrations of acetaminophen and codeine in the supernatant were determined by CD-IMS.

\section{Selectivity study}

The selectivity of the $\mathrm{Fe}_{3} \mathrm{O}_{4} @$ Dual-MIPs was evaluated by the imprinting factor (IF). Morphine, diclofenac, and oxycodone were used as competitive molecules (analogous) in selectivity study. Ten milligrams of $\mathrm{Fe}_{3} \mathrm{O}_{4} @$ Dual-MIPs (or $\mathrm{Fe}_{3} \mathrm{O}_{4} @ \mathrm{NIPs}$ ) were added into a standard solution of analogous compounds with initial concentrations of $20.0 \mu \mathrm{g} \mathrm{mL}^{-1}$. The separation procedure was carried out as described earlier. The imprinting factor was computed from the following equation:

$$
\mathrm{IF}=Q_{\mathrm{MIP}} / Q_{\mathrm{NIP}}
$$

In this equation, $Q_{\mathrm{MIP}}$ and $Q_{\mathrm{NIP}}$ are the adsorption capacity of MIP and NIP, respectively.

\section{Repeatability and reusability of $\mathrm{Fe}_{3} \mathrm{O}_{4} @$ Dual-MIPs}

To evaluate the reusability of $\mathrm{Fe}_{3} \mathrm{O}_{4} @$ Dual-MIPs, 10 mg MIPs and $10 \mathrm{~mL}$ of urine sample, spiked with acetaminophen $\left(20.0 \mu \mathrm{g} \mathrm{mL}^{-1}\right)$ and codeine $\left(15.0 \mu \mathrm{g} \mathrm{mL}^{-1}\right)$, were mixed and shaken, for $30 \mathrm{~min}$. Then, the MIPs were isolated by a magnet and the analytes concentration in the supernatant liquid was determined by IMS. The recovered MIPs were rinsed with methanol-acetic acid $(9: 1, \mathrm{v}: \mathrm{v})$ to eliminate the remaining analytes in the MIPs. The obtained MIPs were rinsed with deionized water and dried in vacuum. These steps were repeated for four cycles of adsorption experiments.

For a repeatability examination, four different batches of $\mathrm{Fe}_{3} \mathrm{O}_{4} @$ Dual-MIPs and $\mathrm{Fe}_{3} \mathrm{O}_{4} @$ NIPs were prepared at different days. $10 \mathrm{mg}$ of each batch was mixed with $10 \mathrm{~mL}$ of an acetaminophen and codeine solution at a concentration of 20 and $15 \mu \mathrm{g} \mathrm{mL}^{-1}$, respectively. After $30 \mathrm{~min}$ of stirring, the supernatant and MIPs were isolated by a magnet and the drugs concentrations were measured by CD-IMS.

\section{Spiked sample analysis}

A drug-free urine sample was used to evaluate the potential of $\mathrm{Fe}_{3} \mathrm{O}_{4} @$ Dual-MIPs for the detection of acetaminophen and codeine. The samples were firstly filtered via a $0.45-\mu \mathrm{m}$ nylon membrane filter (Millipore, Bedford, MA, USA). To minimize the matrix effects, $2 \mathrm{~mL}$ urine sample was diluted till $10 \mathrm{~mL}$ with water (1:5). The urine samples were spiked with acetaminophen and codeine at three various concentration levels $\left(0.5,0.7\right.$, and $\left.1.5 \mu \mathrm{g} \mathrm{mL}^{-1}\right)$. Then, $10 \mathrm{mg}$ of $\mathrm{Fe}_{3} \mathrm{O}_{4} @$ Dual-MIPs or $\mathrm{Fe}_{3} \mathrm{O}_{4} @ \mathrm{NIPs}$ was mixed with $10 \mathrm{~mL}$ of the urine samples containing acetaminophen and codeine. After shaking for $30 \mathrm{~min}$, the $\mathrm{Fe}_{3} \mathrm{O}_{4} @$ Dual-MIPs or $\mathrm{Fe}_{3} \mathrm{O}_{4} @$ NIPs were separated by a magnet and then eluted with $5 \mathrm{~mL}$ of methanol-acetic acid $(9: 1$, v/v) solution. Finally, after evaporation to dryness by nitrogen, the remainder was dissolved in $100 \mu \mathrm{L}$ methanol and determined by CD-IMS.
Also, the proposed method was used for the analysis of acetaminophen and codeine in urine sample from a healthy volunteer after oral administration of an acetaminophen tablet (300 mg) including $10 \mathrm{mg}$ codeine. The samples were obtained, 2 and $5 \mathrm{~h}$ after drug administration.

\section{Results and Discussion}

\section{Preparation of $\mathrm{Fe}_{3} \mathrm{O}_{4} @$ Dual-MIPs}

The synthesis of the $\mathrm{Fe}_{3} \mathrm{O}_{4} @$ Dual-MIPs is a multistep procedure, which involves four steps: (1) Preparation of $\mathrm{Fe}_{3} \mathrm{O}_{4}$ MNPs by a co-precipitation method. (2) Silica-shell deposition $\left(\mathrm{Fe}_{3} \mathrm{O}_{4} @ \mathrm{SiO}_{2}\right)$ using TEOS by a sol-gel process. This modification provides good biocompatibility and decreases the magnetic dipole attraction between the magnetite nanoparticles, preventing aggregation in liquid. (3) The interaction between template molecules and monomers and then polymerization by using cross-linker. The combination of monomer-template was achieved with acetaminophen and codeine as templates and monomers (APTES and PTEOS). Then, the templatesmonomers complex was attached on the surface of $\mathrm{Fe}_{3} \mathrm{O}_{4} @ \mathrm{SiO}_{2}$ in presence of ammonium hydroxide as the catalyst and TEOS as a cross-linker by sol-gel process. (4) Finally, the templates were removed to obtain the $\mathrm{Fe}_{3} \mathrm{O}_{4} @$ Dual-MIPs and create imprinted cavities for specific diagnosis of studied analytes.

The amounts of monomers and template molecules affect the sorbent capacity as well as the recognition capability of the MIPs. Therefore, the volume ratios of APTES and PTEOS and the amount of templates and monomers were evaluated, the results of which are given in Table S2 (Supporting Information). In order to obtain the maximum $Q$ and IF values, the optimized conditions of polymerization were selected. Based on the results, the optimum ratio of functional monomers APTES/ PTEOS was $1: 2$ and the optimum amount of templates and functional monomers were $20 \mathrm{mg}, 110$ and $220 \mu \mathrm{L}$, respectively. In this work, $\mathrm{Fe}_{3} \mathrm{O}_{4} @$ Dual-MIPs showed a good adsorption capability related to acetaminophen and codeine, likely due to hydrogen bonds and $\pi-\pi$ interactions. One of the functional monomers is APTES that may establish hydrogen bonds between the amino and hydroxyl groups of the template molecules. PTEOS is the other functional monomer, may conduct the $\pi-\pi$ interactions with the templates via the phenyl groups.

\section{MMIPs characterization}

The FTIR spectra of $\mathrm{Fe}_{3} \mathrm{O}_{4}, \mathrm{Fe}_{3} \mathrm{O}_{4} @ \mathrm{SiO}_{2}$, and $\mathrm{Fe}_{3} \mathrm{O}_{4} @$ DualMIPs are given in Fig. S1 (A, B, and C), in the Supporting Information. According to this figure, the band at $58 \mathrm{~cm}^{-1}$ corresponded to vibrations of the $\mathrm{Fe}-\mathrm{O}$ bonds. The absorption bands at 1640 and $3420 \mathrm{~cm}^{-1}$ were assigned to bending and stretching vibrations of $\mathrm{O}-\mathrm{H}$, respectively. In addition, the absorption bands at 800, 949, and $1100 \mathrm{~cm}^{-1}$ (in Figs. S1B and $\mathrm{S} 1 \mathrm{C})$ were attributed to the stretching vibrations of $\mathrm{Si}-\mathrm{O}$, $\mathrm{Si}-\mathrm{O}-\mathrm{H}$, and $\mathrm{Si}-\mathrm{O}-\mathrm{Si}$, respectively, indicating the successful synthesis of $\mathrm{Fe}_{3} \mathrm{O}_{4} @ \mathrm{SiO}_{2}$. The existence of characteristic bands at 1562 and $1430 \mathrm{~cm}^{-1}$ corresponded to the bending vibration of $\mathrm{N}-\mathrm{H}$ and stretching vibration of $\mathrm{Si}-\mathrm{C}_{6} \mathrm{H}_{5}$, originated from APTES and PTEOS, respectively (Fig. S1C).

The TEM images obtained for $\mathrm{Fe}_{3} \mathrm{O}_{4}$ and $\mathrm{Fe}_{3} \mathrm{O}_{4} @$ Dual-MIPs are given in Fig. 1. This figure shows that the prepared nanomaterials represented the almost spherical shape. According to Fig. $1 \mathrm{~A}$, the pure $\mathrm{Fe}_{3} \mathrm{O}_{4}$ nanoparticles were relatively homogeneous with an average diameter about $20 \mathrm{~nm}$. As can be observed in Fig. 1B, the average diameter of $\mathrm{Fe}_{3} \mathrm{O}_{4} @$ Dual-MIPs 

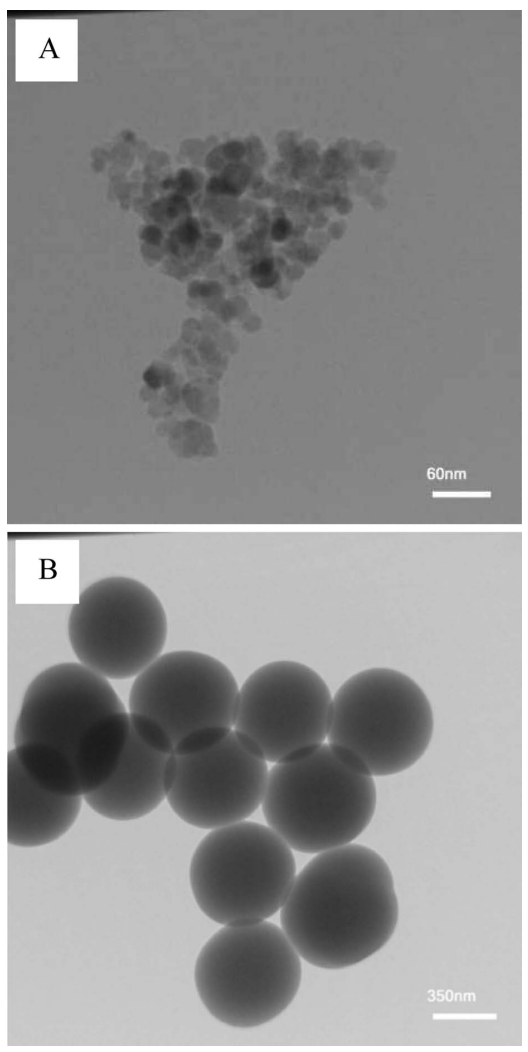

Fig. 1 TEM image of $\mathrm{Fe}_{3} \mathrm{O}_{4}(\mathrm{~A})$ and $\mathrm{Fe}_{3} \mathrm{O}_{4} @$ Dual-MIPs (B).

increased to about $580 \mathrm{~nm}$ after the imprinting process, indicating the formation of MIP on the nanoparticles' surface.

Additionally, the XRD instrument was used to characterize the crystal structure. Figure S2 (Supporting Information) illustrates the XRD pattern for $\mathrm{Fe}_{3} \mathrm{O}_{4}(\mathrm{~A}), \mathrm{Fe}_{3} \mathrm{O}_{4} @ \mathrm{SiO}_{2}(\mathrm{~B})$, and $\mathrm{Fe}_{3} \mathrm{O}_{4} @$ Dual-MIPs (C). The XRD patterns of pure $\mathrm{Fe}_{3} \mathrm{O}_{4}$ and modified $\mathrm{Fe}_{3} \mathrm{O}_{4}$ showed six characteristic peaks $(2 \theta=30.2$, 35.6, 43.1, 53.7, 57.2, and $62.9^{\circ}$ ). These results indicate that no changing had occurred in the obtained magnetite nanoparticles, during the preparation of $\mathrm{Fe}_{3} \mathrm{O}_{4} @$ Dual-MIPs.

\section{Kinetics of adsorption}

Figure 2 depicts the adsorption kinetics of acetaminophen and codeine onto the MIP particles. This is the typical kinetic curve for most rebinding processes, indicating a rapid dynamic adsorption of acetaminophen and codeine to the magnetic MIPs. As is clear in this figure, there was a fast increasing of adsorption in the first $30 \mathrm{~min}$ before equilibrium. MIPs provided an efficient mass transfer due to the thin and uniform imprinted films; so template molecules could approach the recognition sites of $\mathrm{Fe}_{3} \mathrm{O}_{4} @$ Dual-MIPs and take a short time to reach the equilibrium of adsorption. These results reveal that binding sites of the MIPs were formed on or near the surface.

\section{Adsorption isotherm}

The adsorption isotherms curve is one of the methods for investigating the thermodynamic adsorption properties of the MIPs (as shown in Fig. S3, Supporting Information). The experiments were conducted with different initial concentrations of acetaminophen and codeine ranging from 5.0 to 35.0 and 5.0 to $30.0 \mu \mathrm{g} \mathrm{mL}^{-1}$, respectively. The adsorption capacity of MIP was continuously increased with the raising the initial concentration of acetaminophen and codeine and reached the

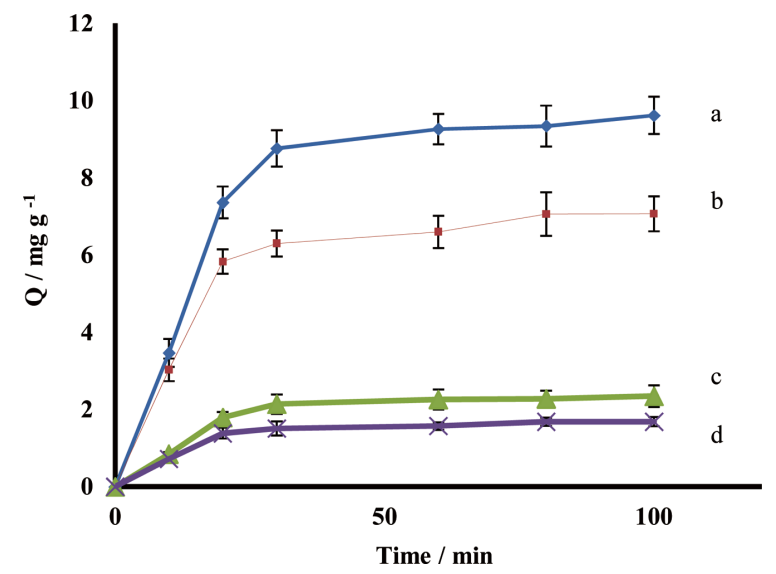

Fig. 2 Adsorption kinetics of $\mathrm{Fe}_{3} \mathrm{O}_{4} @$ Dual-MIPs and $\mathrm{Fe}_{3} \mathrm{O}_{4} @$ NIPs to acetaminophen ( $\mathrm{a}$ and $\mathrm{c}$ ) and codeine ( $\mathrm{b}$ and $\mathrm{d}$ ), respectively.

equilibrium at 20.0 and $15.0 \mu \mathrm{g} \mathrm{mL}^{-1}$, respectively. Based on these results, at the same initial concentration, the amounts of acetaminophen and codeine bound to $\mathrm{Fe}_{3} \mathrm{O}_{4} @$ Dual-MIPs were higher than that of $\mathrm{Fe}_{3} \mathrm{O}_{4} @$ NIPs. This revealed the important role of imprinted recognition sites in the process of analytes binding to $\mathrm{Fe}_{3} \mathrm{O}_{4} @$ Dual-MIPs. The Langmuir model was used to process the adsorption data which the follow equation could be explained:

$$
\frac{C_{\mathrm{e}}}{Q_{\mathrm{e}}}=\frac{C_{\mathrm{e}}}{Q_{\max }}+\frac{1}{K_{\mathrm{L}} Q_{\max }},
$$

where, $C_{\mathrm{e}}\left(\mu \mathrm{g} \mathrm{mL}^{-1}\right)$ is the equilibrium concentration of templates, $K_{\mathrm{L}}$ is the adsorption constant, and $Q_{\mathrm{e}}$ and $Q_{\max }$ $\left(\mathrm{mg} \mathrm{g}^{-1}\right)$ are the saturated and the maximum adsorption capacity values, respectively. As shown in Fig. S4 (Supporting Information), the plot of $C_{\mathrm{e}} / Q_{\mathrm{e}}$ versus $C_{\mathrm{e}}$ is linear. The Langmuir isothermal plots showed $R^{2}$ values of 0.995 and 0.993 for acetaminophen and codeine, respectively. From the obtained straight lines, the $Q_{\max }$ of acetaminophen and codeine were calculated to be 9.90 and $7.24 \mathrm{mg} \mathrm{g}^{-1}$ for $\mathrm{Fe}_{3} \mathrm{O}_{4} @$ Dual-MIPs, and 2.55 and $1.83 \mathrm{mg} \mathrm{g}^{-1}$ for $\mathrm{Fe}_{3} \mathrm{O}_{4} @$ NIPs. These results are close to the maximum amounts of adsorption obtained experimentally (Fig. S3). This indicates that the adsorption of template molecules by $\mathrm{Fe}_{3} \mathrm{O}_{4} @$ Dual-MIPs is dependent on to the monolayer absorption and so the surface is uniform.

\section{Specific recognition}

In order to evaluate the specificity of $\mathrm{Fe}_{3} \mathrm{O}_{4} @$ Dual-MIPs for acetaminophen and codeine, some experiments were conducted by selecting morphine, diclofenac and oxycodone as comparative molecules (analogues). The obtained results are shown in Fig. S5 (Supporting Information). The selectivity coefficient (SC) and imprinting factor (IF) were calculated to show the specific recognition property of the MIPs related to template molecules and comparative molecules. The SC value was computed by

$$
\mathrm{SC}=\mathrm{IF}_{\mathrm{t}} / \mathrm{IF}_{\mathrm{a}},
$$

where, $\mathrm{IF}_{\mathrm{t}}$ and $\mathrm{IF}_{\mathrm{a}}$ are the imprinting factors for template and analogue molecules. According to the obtained results, the imprinted polymers presented much higher binding amounts $\left(Q_{\text {MIP }}\right)$ for acetaminophen and codeine $\left(8.76\right.$ and $\left.6.29 \mathrm{mg} \mathrm{g}^{-1}\right)$ in comparison with those of morphine, diclofenac and oxycodone 
Table 1 Adsorption capacities, imprinting factors, and selectivity coefficient of templates and analogues for $\mathrm{Fe}_{3} \mathrm{O}_{4} @$ Dual-MIPs and $\mathrm{Fe}_{3} \mathrm{O}_{4} @ \mathrm{NIPs}$

\begin{tabular}{lccccc}
\hline \multicolumn{1}{c}{ Analyte } & $Q_{\mathrm{MIP}}$ & $Q_{\mathrm{NIP}}$ & $\mathrm{IF}$ & $\mathrm{SC}_{\text {Acet }}$ & $\mathrm{SC}_{\mathrm{Cod}}$ \\
\hline Acetaminophen & 8.76 & 2.14 & 4.09 & - & - \\
Codeine & 6.29 & 1.51 & 4.16 & - & - \\
Morphine & 1.64 & 1.02 & 1.61 & 2.54 & 2.58 \\
Oxycodone & 2.48 & 1.86 & 1.33 & 3.07 & 3.13 \\
Diclofenac & 2.12 & 1.43 & 1.48 & 2.76 & 2.81 \\
\hline
\end{tabular}

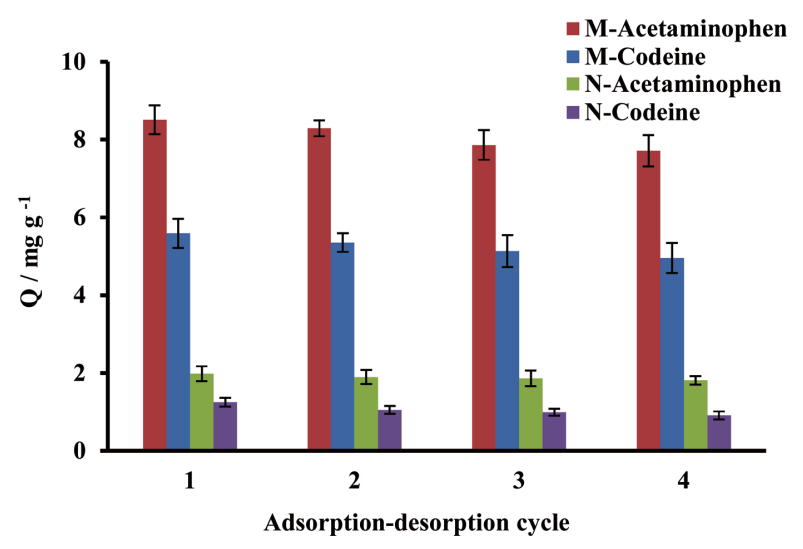

Fig. 3 Reusability of $\mathrm{Fe}_{3} \mathrm{O}_{4} @$ Dual-MIPs and $\mathrm{Fe}_{3} \mathrm{O}_{4} @$ NIPs.

(1.64, 2.12 and $2.48 \mathrm{mg} \mathrm{g}^{-1}$, respectively). These results indicate that the specific sites existing in magnetic MIP may be supplementary in shape, size, and spatial distribution, as well as functional groups, corresponded to template molecules. Since the adsorption process of magnetic NIP was nonspecific, the magnetic NIP exhibited similar selectivity for templates and other analogues molecules. As tabulated in Table 1, the $\mathrm{Fe}_{3} \mathrm{O}_{4} @$ Dual-MIPs represented the significant adsorption specificity for acetaminophen and codeine with IF and SC values above 4.09 and 2.54 , respectively. These results approved the satisfactory imprinting efficiency obtained by the present method.

\section{Repeatability and reusability}

The repeatability of the prepared MMIPs was studied using four batches of $\mathrm{Fe}_{3} \mathrm{O}_{4} @$ NIPs and $\mathrm{Fe}_{3} \mathrm{O}_{4} @$ Dual-MIPs at different days. For calculating the standard deviation, three replicated measurements were conducted for each batch. The average adsorption capacity of the templates was calculated for each batch, and the results are tabulated in Table S3 (in Supporting Information). Statistical analysis was done on data by analysis of variance (ANOVA) test. The results show that the RSD values are less than $7.8 \%$, reveal the acceptable repeatability for all the batches of $\mathrm{Fe}_{3} \mathrm{O}_{4} @$ Dual-MIPs and $\mathrm{Fe}_{3} \mathrm{O}_{4} @$ NIPs.

To verify the reusability of $\mathrm{Fe}_{3} \mathrm{O}_{4} @$ Dual-MIPs, different adsorption/desorption cycles were repeated four times using the same $\mathrm{Fe}_{3} \mathrm{O}_{4} @$ Dual-MIPs and $\mathrm{Fe}_{3} \mathrm{O}_{4} @$ NIPs. The results are depicted in Fig. 3, indicating that the $\mathrm{Fe}_{3} \mathrm{O}_{4} @$ Dual-MIPs could be used for four cycles, at least. The adsorption capacities in first and fourth cycles are 8.53 and 7.71 for acetaminophen and 5.58 and 4.95 , for codeine. So, the adsorption capacities remained at steady values of 90.38 and $88.71 \%$ for acetaminophen and codeine, respectively. Consequently, the reusability experiments of $\mathrm{Fe}_{3} \mathrm{O}_{4} @$ Dual-MIPs and $\mathrm{Fe}_{3} \mathrm{O}_{4} @$ NIPs confirmed the desirable stability of the prepared MMIPs.

\section{Analytical parameters}

In order to perform quantitative analysis, a series of standards in the concentration range of $0.2-2.0 \mu \mathrm{g} \mathrm{mL}^{-1}$ for acetaminophen and $0.4-3.0 \mu \mathrm{g} \mathrm{mL}^{-1}$ for codeine were prepared in pure methanol. To draw calibration curves, the response of the CD-IMS was plotted versus the concentration, also, the area of the peak was considered as a response of the instrument. The limit of detection (LODs) and the limit of quantitation (LOQs), were assumed as LOD $=3 S_{\mathrm{b}} / m$, and LOQ $=10 S_{\mathrm{b}} / m$. Herein, $m$, $S_{\mathrm{b}}$ and are the slope of the calibration graph, and the standard deviations of the blank, respectively. In this study, LODs and LOQs were obtained 0.05 and $0.20 \mu \mathrm{g} \mathrm{mL}^{-1}$ for acetaminophen and 0.12 and $0.40 \mu \mathrm{g} \mathrm{mL}^{-1}$ for codeine. However, in order to study the quantitative analysis possibility for two compounds in the binary mixture, it is necessary to investigate the analyte interference. To this end, the slopes of the calibration curves for a series of acetaminophen solutions and the same series of acetaminophen solutions in the presence of a constant amount of codeine were compared with each other. This comparison was also conducted for the calibration curves of codeine in the absence or presence of acetaminophen. The results are shown in Fig. S6 (Supporting Information). The results show that acetaminophen and codeine did not show analyte interference on the measurement of another one.

\section{Spiked sample analysis}

The capability of $\mathrm{Fe}_{3} \mathrm{O}_{4} @$ Dual-MIPs were investigated by spiking the urine samples with acetaminophen and codeine at concentration levels of $0.5,0.7$, and $1.5 \mu \mathrm{g} \mathrm{mL} \mathrm{m}^{-1}$. For each concentration, three measurements were conducted. Table S4 (Supporting Information) represents the IMS results obtained for the analytes extracted from urine samples by $\mathrm{Fe}_{3} \mathrm{O}_{4} @$ DualMIPs. Figure S7 (Supporting Information) depicts the ion moility spectrum obtained after the direct injection of diluted urine (5 times) into the apparatus. In addition, the ion mobility spectra obtained for extracted samples from the urine samples spiked with acetaminophen and codeine (both $1.5 \mu \mathrm{g} \mathrm{mL}^{-1}$ ), compared with that obtained for the unspiked urine samples (blank), are shown in Fig. S7. According to these mobility spectra, it is observed that different impurities in the urine sample could be eliminated using MIPs due to the selectivity property of these sorbents. This resulted in the analysis of acetaminophen and codeine, without any interference of matrix interference. In fact, the extraction and cleanup steps for the urine samples can greatly reduce the matrix interference and provide the satisfactory conditions for analytes analysis by $\mathrm{CD}$ IMS. The results showed that the MIP extraction of acetaminophen and codeine for urine samples have good precision (RSD lower than $8 \%$ ) with the recoveries between 87 and 94 percent. Therefore, $\mathrm{Fe}_{3} \mathrm{O}_{4} @$ Dual-MIPs combined with CD-IMS can be used as a reliable technique for the trace analysis of acetaminophen and codeine, simultaneously, in the biological samples.

In order to evaluate the method validation, a standard method based on HPLC instrument with UV detection ${ }^{40}$ was also used. In this regard, the analytes in the urine of healthy volunteers were analyzed, after the oral administration of drug tablet (containing $300 \mathrm{mg}$ acetaminophen/10 mg codeine). Quantification of acetaminophen and codeine in the real sample was performed by single standard addition method. The ion mobility spectrum of the extracted sample is shown in Fig. 4. The analytical results of CD-IMS were compared with those obtained by HPLC method which the statistical results is tabulated in Table 2. These results indicate the proposed method capability in simultaneous analyses of the analyte in real samples. 


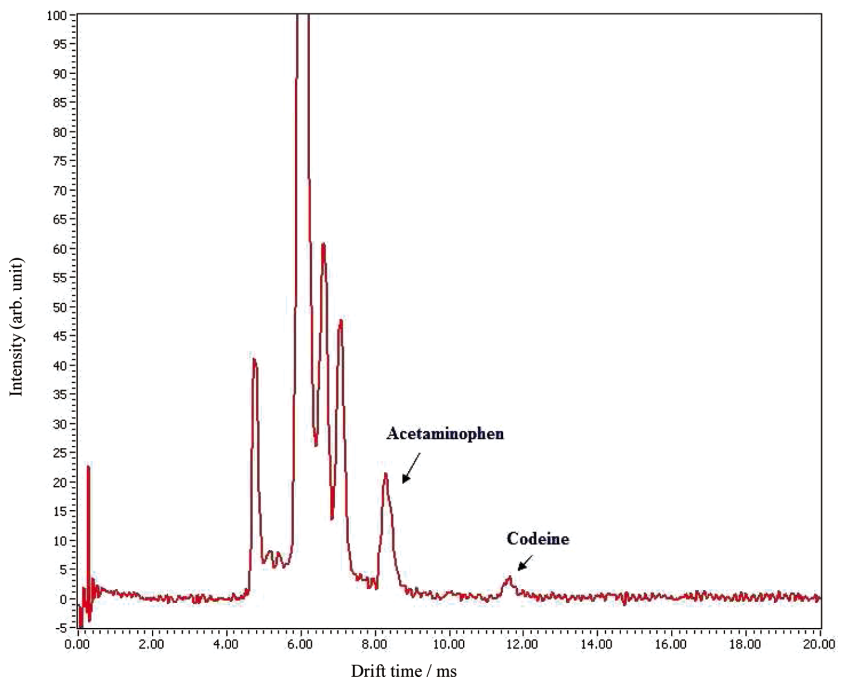

Fig. 4 Ion mobility spectrum of the extracted sample from urine, $2 \mathrm{~h}$ after drug administration.

Table 2 Comparison of CD-IMS and HPLC in the analysis of acetaminophen and codeine in urine samples obtained from volunteer after oral administration of a 300-mg acetaminophen tablet containing $10 \mathrm{mg}$ codeine (the statistical results reveal the accuracy of the CD-IMS method for simultaneous analysis of the analytes)

\begin{tabular}{|c|c|c|c|c|c|c|}
\hline \multirow{2}{*}{ Analyte } & \multirow{2}{*}{$\begin{array}{c}\text { Sampling } \\
\text { time after } \\
\text { drug } \\
\text { taking/h }\end{array}$} & \multicolumn{2}{|c|}{$\begin{array}{l}\text { Found amount/ } \\
\qquad \mu \mathrm{g} \mathrm{mL}^{-1}\end{array}$} & \multirow{2}{*}{$S_{\text {pooled }^{\mathrm{a}}}$} & \multirow{2}{*}{$t_{\text {exp. }}{ }^{\mathrm{b}}$} & \multirow{2}{*}{$\begin{array}{c}t_{\text {tab. }} \\
(95 \%)^{\mathrm{c}}\end{array}$} \\
\hline & & $\begin{array}{l}\text { CD- } \\
\text { IMS }\end{array}$ & $\begin{array}{l}\text { HPLC- } \\
\text { UV }\end{array}$ & & & \\
\hline Acet & 2 & 3.01 & 3.53 & 0.56 & 1.13 & 2.78 \\
\hline Cod & 2 & 1.21 & 0.97 & 0.35 & 0.84 & 2.78 \\
\hline Acet & 5 & 4.05 & 4.35 & 0.68 & 0.54 & 2.78 \\
\hline Cod & 5 & 3.14 & 3.58 & 0.52 & 1.03 & 2.78 \\
\hline
\end{tabular}

a. The pooled value of the standard deviation.

b. The experimental value of $t$.

c. The critical value of $t$.

\section{Conclusions}

In this study, we prepared magnetic dual-template molecularly imprinted nanoparticles by coating thin shells of functional monomer (APTES and PTEOS) onto the surface of silica-coated MNPs. This sorbent was used for the extraction and clean-up the acetaminophen and codeine compounds (as templates) from the urine samples. The ion mobility spectrometry, IMS, was used as the determination system for qualitative and quantitative analysis of the analytes, simultaneously. The advantages of the MMIP preparation were the sol-gel approach, surface imprinting technique, and magnetic separation for the specific adsorption of acetaminophen and codeine, simultaneously. Resultant product represented a fast adsorption, large binding capacity, good selectivity and satisfactory repeatability and reusability for two templates. Furthermore, the $\mathrm{Fe}_{3} \mathrm{O}_{4} @$ Dual-MIPs combined with IMS has been favorably used as useful technique for the separation and determination of acetaminophen and codeine in urine samples without any tedious sample preparation steps such as analyte derivatization. The satisfactory qualitative results obtained in this work reveal the capability of this combined method, for routine analysis of acetaminophen and codeine in various biological matrices.

\section{Acknowledgements}

The authors acknowledge Isfahan University of Technology and the Research Council and Center of Excellence in Sensor and Green Chemistry of IUT for supporting this work.

\section{Supporting Information}

FT-IR spectra and XRD patterns of $\mathrm{Fe}_{3} \mathrm{O}_{4}, \mathrm{Fe}_{3} \mathrm{O}_{4} @ \mathrm{SiO}_{2}$ and $\mathrm{Fe}_{3} \mathrm{O}_{4} @$ Dual-MIPs were prepared. Adsorption isotherms and langmuir isothermal plots were given. The specific adsorption capability of $\mathrm{Fe}_{3} \mathrm{O}_{4} @$ Dual-MIPs and $\mathrm{Fe}_{3} \mathrm{O}_{4} @ \mathrm{NIPs}$ were provided. Information about IMS operation parameters, optimization of functional monomers and templates amounts, repeatability and analysis of real sample were presented in Supporting Information. These materials are available free of charge on the Web at http://www.jsac.or.jp/analsci/.

\section{References}

1. R. Chandra and K. D. Sharma, Int. J. Chromatogr. Sci., 2013, 3, 31 .

2. V. Maslarska and J. Tencheva, Int. J. Pharm. Pharm. Sci., 2013, 5, 417.

3. J. G. Bessems and N. P. Vermeulen, Crit. Rev. Toxicol., 2001, 31, 55.

4. M. Shahlaei, H. Andisheh, K. Derakhshandeh, K. S. Havadi, and M. Azami, J. Rep. Pharm. Sci., 2014, 3, 141.

5. V. N. Edebi, B. U. Ebeshi, and E. Anganabiri, Afr. J. Pure Appl. Chem., 2011, 5, 104.

6. J. M. Garrido, C. Delerue-Matos, F. Borges, T. R. Macedo, and A. M. Oliveira-Brett, Anal. Lett., 2002, 35, 2487.

7. L. Toms, S. Derry, R. A. Moore, and H. J. McQuay, Cochrane Libr., 2009, 1, 1

8. M. Mazer and J. Perrone, J. Med. Toxicol., 2008, 4, 2.

9. A. K. Hameed, A. J. Hussein, and S. K. Majeed, Bas. J. Vet. Res., 2015, 14, 167.

10. A. H. Schmidt, J. Liq. Chromatogr. Relat. Technol., 2006, 29, 1663.

11. J. Hanaee, Pharm. Acta Helv., 1997, 72, 239.

12. D. T. T. An and V. D. Hoang, Asian J. Res. Chem., 2009, 2, 143.

13. A. Mokhtari, N. Jafari Delouei, M. Keyvanfard, and M. Abdolhosseini, Luminescence, 2016, 13, 1267.

14. P. Batista Deroco, F. Campanhã Vicentini, and O. Fatibello Filho, Electroanalysis, 2015, 27, 2214.

15. E. Garazhian and M. R. Shishehbore, Int. J. Anal. Chem., 2015, 2015, 1.

16. A. A. Ensafi, N. Ahmadi, B. Rezaei, and M. M. Abarghoui, Talanta, 2015, 134, 745.

17. X. Chen, J. Rao, J. Wang, J. J. Gooding, G. Zou, and Q. Zhang, Chem. Commun., 2011, 47, 10317.

18. P. Fernandez, C. Vazquez, L. Morales, and A. M. Bermejo, J. Appl. Toxicol., 2005, 25, 200.

19. T. Madrakian, F. Fazl, M. Ahmadi, and A. Afkhami, New J. Chem., 2016, 40, 122.

20. G. Wulff, A. Sarhan, and K. Zabrocki, Tetrahedron Lett., 1973, 14, 4329.

21. G. Vasapollo, R. D. Sole, L. Mergola, M. R. Lazzoi, 
A. Scardino, S. Scorrano, and G. Mele, Int. J. Mol. Sci., 2011, 14, 5908.

22. A. Kugimiya and T. Takeuchi, Anal. Sci., 1999, 15, 29.

23. Y. H. Li, T. Yang, X. L. Qi, Y. W. Qiao, and A. P. Deng, Anal. Chim. Acta, 2008, 624, 317.

24. S. Song, X. Shi, R. Li, Z. Lin, A. Wu, and D. Zhang, Process Biochem., 2008, 43, 1209.

25. X. Kan, Y. Zhao, Z. Geng, Z. Wang, and J. J. Zhu, J. Phys. Chem. C, 2008, 112, 4849 .

26. J. Ma, L. Yuan, M. Ding, S. Wang, F. Ren, J. Zhang, S. Du, F. Li, and X. Zhou, Biosens. Bioelectron., 2011, 26, 2791.

27. F. Barahona, E. Turiel, and A. Martín-Esteban, J. Chromatogr. A, 2011, 1218, 7065.

28. F. Deng, Y. Li, X. Luo, L. Yang, and X. Tu, Colloids Surf., A, 2012, 395, 183.

29. X. Wang, L. Wang, X. He, Y. Zhang, and L. Chen, Talanta, 2009, 78, 327.

30. M. Feng, H. Li, L. Zhang, J. Zhang, J. Dai, X. Wang, L. Zhang, and Y. Wei, Anal. Sci., 2016, 32, 517.
31. L. Chen, J. Liu, Q. Zeng, H. Wang, A. Yu, H. Zhang, and L. Ding, J. Chromatogr. A, 2009, 1216, 3710.

32. M. T. Jafari, B. Rezaei, and B. Zaker, Anal. Chem., 2009, 81,3585

33. M. T. Jafari, Z. Badihi, and E. Jazan, Talanta, 2012, 99, 520.

34. B. Rezaei, M. T. Jafari, and O. Rahmanian, Talanta, 2011 , 83,765

35. B. Rezaei, M. T. Jafari, and R. Khademi, Talanta, 2009, 79, 669.

36. A. Sorribes-Soriano, F. A. Esteve-Turrillas, S. Armenta, M. de la Guardia, and J. M. Herrero-Martínez, J. Chromatogr. A, 2017, 1481, 23

37. G. A. Eiceman, Z. Karpas, and H. H. Hill Jr, "Ion Mobility Spectrometry", 3rd ed., 2014, CRC Press, Boca Raton.

38. M. T. Jafari and F. Riahi, J. Chromatogr. A, 2014, 1343, 63.

39. R. Gao, Y. Hao, S. Zhao, L. Zhang, X. Cui, D. Liu, Y. Tang, and Y. Zhenga, RSC Adv., 2014, 100, 56798.

40. V. D. Gupta, J. Pharm. Sci., 1980, 69, 110. 